\title{
Impact of chronic pain on health care seeking, self care, and medication. Results from a population-based Swedish study
}

\author{
H Ingemar Andersson, Göran Ejlertsson, Ido Leden, Bengt Scherstén
}

\begin{abstract}
Study objective-To explore individual and social factors that could predict health care utilisation and medication among people with chronic pain in an unselected population.
\end{abstract}

Design-A mailed survey with questions about pain and mental symptoms, disability, self care action, visits to health care providers, and medication.

Setting-General populations in two Swedish primary health care (PHC) districts. Medical care was given in a state health system.

Participants-A random sample (from the population register) of $15 \%$ of the population aged 25-74 $(n=1806)$.

Main results-Among people reporting chronic pain $45.7 \%$ (compared with 29.8 of non-chronic pain persons, $p<0.05)$ consulted a physician and $7.2 \%$ (compared with $1.2 \%, p<0.05$ ) a physiotherapist during three months. Primary health care was the most frequent care provider. High pain intensity, aging, depression, ethnicity, and socioeconomic level had the greatest impact on physician consultations. Alternative care, used by $5.9 \%$, was associated with high pain intensity and self care. Use of self care was influenced by high pain intensity, regular physical activity, and ethnicity. Alternative care and self care did not imply lower use of conventional health care. Women reporting chronic pain consumed more analgesics and sedatives than corresponding men. Besides female gender, high pain intensity, insomnia, physician consultation, social network, and self care action helped to explain medication with analgesics. Use of herbal remedies and ointments correlated to self care action, visit to an alternative therapist, high pain intensity, and socioeconomic level.

Conclusions-The presence of chronic pain has an impressive impact on primary health care and medication. Various therapeutic actions are common and are partly overlapping. The use of health care among people with chronic pain depends above all on pain perception and intensity of pain but is also affected by ethnicity, age, socioeconomic level, and depressive symptoms. Among people with chronic pain use of analgesics is common in contrast with other types of pain relief (acupuncture, physiotherapy) suitable for treating chronic pain symptoms.

(F Epidemiol Community Health 1999;53:503-509)
Chronic pain (duration more than three months) is a common problem among people in different societies. ${ }^{1-3}$ The genesis of the pain symptoms is multifactorial depending on individual physical and psychosomatic factors as well as socioeconomic and other interactions with the society. ${ }^{4}$ Population studies have confirmed obvious inequality of pain prevalence between different groups of the community. Age, gender, socioeconomic level, and ethnicity all affect the frequency of pain symptoms. ${ }^{15}$

Studies from primary health care suggest that chronic pains especially from the musculoskeletal system are a frequent reason for consultation. ${ }^{67}$ Care seeking because of chronic pain depends on individual and social factors and is only partly understood. Despite the fact that chronic pain has an important impact on health and the insurance system, only few studies have focused on chronic pain and utilisation of health care. Severity, persistence, and recency of the onset of pain have been shown to be determinants for seeking care for specific pain symptoms. ${ }^{7}$ Other studies have pointed out a general influence on the consultation rate from age, gender, ethnicity, and psychological factors.

In studies of ambulatory visits women have, for most diagnoses including musculoskeletal diseases, more health care contacts than men. ${ }^{8}$ Women have also been attributed a higher consumption of analgesics and use of alternative remedies. ${ }^{9}$ Epidemiological studies of chronic pain, mostly of musculoskeletal origin, have demonstrated different distributions by gender. Some studies show a general preponderance of women reporting pain symptoms ${ }^{37}$ while other studies ${ }^{12}$ report no difference by gender in total pain prevalence. The diverging results may reflect real differences in populations or methodological differences. However, the discrepancy of pain reports by gender and care seeking pattern needs a closer examination.

Outside the established health care system several health care providers offer chronic pain patients cure with a variety of methods. Pain problems have been displayed to be important reasons for seeking alternative medicine. ${ }^{10}$ The extent of alternative health care and self care in relation to contacts with established medicine and prevalence of chronic pain has been rarely studied.

The broad uses of analgesics as pain relief has been especially studied in aggregated form by means of prescription data and pharmacy sales statistics. The relation between diagnoses and prescribed drugs in Sweden has been 
recurrently analysed by National Corporation of Swedish Pharmacy ${ }^{11}$ but these data do not comprise unprescribed medication. A Swedish population study deals with the consumption of both prescribed and unprescribed analgesics $^{12}$ and emphasises that women compared with men use more drugs and analgesics, but these data are not related to the type of pain problem.

The aims of this study were to evaluate the amount of self care and health care, both established and alternative, used by people with chronic pain in society, and the use of different medications for pain relief. We also wanted to evaluate to what extent individual and social factors could predict consulting rates and levels of self care and medication. The Swedish health care system with a uniform organisation of health providers, mainly financed by the state, and a well defined population register constitutes a suitable setting for studies of health care and medication.

\section{Methods}

Data on pain reports and measures taken to get pain relief (health care contacts, self therapy, and medication) were collected as a part of an epidemiological study of chronic pain, described earlier. ${ }^{1}$ A mailed questionnaire was sent to a random sample $(\mathrm{n}=1806)$ of persons aged $25-74$ in two primary health care (PHC) districts in the south of Sweden. The response rate was $89 \%$. The questionnaire included questions about pain duration, location and intensity (graded in five steps $1-5$, where high pain intensity was defined as grade $4-5$ ). Other reports of illness, including both somatic and psychiatric symptoms during the past three months, were captured with a standard symptom list. ${ }^{1}$

Activities of Daily Living (ADL) were rated by graded questions about the ability to perform seven different activities (walk, shop, eat, dress, wash, manage stairs, and go to the toilet). The ability was rated according to three grades: no difficulties, some difficulties, and great difficulties, and it was emphasised that the impairment should be attributable to pain. Reduced ADL capacity was specified as some or great difficulty in any of the activites.

Health care utilisation was estimated from questions about consultations with different types of physicians (primary health care, occupational health care, hospital care), physiotherapist, acupuncturist, chiropractor, homeopathist, and practiser of naturopathy. Contacts during the past three months were graded in four steps: no contact, once, 2-5 times and more than five times. For numerical comparisons of contacts a calculated sum of visits was obtained by using the numbers 0,1 , 3 , and 6 respectively to represent each of the steps mentioned above.

Socioeconomic level was grouped according to a method used by Statistics Sweden, mainly based on trade union affiliation. ${ }^{13}$ We used four levels of education, from comprehensive school (8-9 years) to university. An immigrant was defined as a settler coming to Sweden, or both parents being immigrants. Data were captured by the question "Have you or both of your parents immigrated to Sweden?" The immigrants, mainly from Mediterranean countries, constituted $7.2 \%$ of the respondents.

Physical work strain was reported according to five pretested questions and thus graded 0 to 5 , where 0 implied no physical work strain and 5 the highest physical work strain. ${ }^{13}$

A social network was graded as an index $(0-7$ from the lowest $=0$ to the most intense $=7$ ) from the answers to four questions about organisational activities, of intensity of contacts, intimacy of contacts, and social support. The questions related to a Swedish study of social network. ${ }^{14}$ The index score was dichotomised and a score above four was defined as an intense social network, the score $0-4$ as a low social network.

Report of sickness absenteeism (including the number of days) attributable to any pain symptom during the past three months was collected by two questions. ${ }^{13}$

Consumption of drugs was assessed from questions about the intake of analgesics (divided into three groups: aspirin/ paracetamol, non-steroidal anti-inflammatory drugs (NSAID)/combined analgesics and combined muscle relaxants), tranquillisers, hypnotics, ointments, and natural medicine during a 14 day period before the survey. The amount of medication was graded in six steps: several times a day, daily, several times a week, weekly, more seldom and never. These questions were used and validated earlier. ${ }^{13}$

SETTING

The studied population lives in two PHC districts in the south of Sweden, Bromölla and Simrishamn.

Bromölla Health Centre serves the inhabitants of a well defined rural district (12 500 inhabitants) with primary health care since the beginning of the 1960s. Besides district physicians and nurses, physiotherapists and occupational therapists work at the health centre. Secondary care is available at a Central Hospital 25 kilometres west of Bromölla.

Simrishamn is a small town at the seaside (8800 inhabitants) where trade, fishing, and farming are important occupations. The local hospital houses the health centre with district physicians and also nurses, physiotherapists, and occupational therapists. Secondary care is also provided at the hospital. Hardly any physiotherapist at the time of the study used acupuncture, but had the opportunity to try out transcutaneous electrical nerve stimulation.

As results from the epidemiological study ${ }^{1}$ of pain did not differ between the two locations and in view of the great similarity of health care organisation in this study we treated the results from both settings together.

STATISTICAL METHODS

A $\chi^{2}$ test was used to compare the report of self care, different care contacts, and medication between groups. When a stratified analysis was performed (comparison of calculated number of contacts) the Mantel-Haenszel $\chi^{2}$ test for 
Table 1 Comparison of vistors and non-visitors to PHC in the past three month among people reporting chronic pain (duration $>3$ months) with a high pain intensity (4-5 on a 5 step scale)

\begin{tabular}{llll}
\hline & $\begin{array}{l}\text { Visitors } \\
(n=225)\end{array}$ & $\begin{array}{l}\text { Non-visitors } \\
(n=174)\end{array}$ & $p$ \\
\hline Age (median, y) & 51.0 & 49.5 & $\mathrm{NS}$ \\
Female gender (\%) & 55.1 & 50.6 & $\mathrm{NS}$ \\
Education >9 years (\%) & 12.0 & 8.6 & $\mathrm{NS}$ \\
$\begin{array}{l}\text { Socioeconomic level (\%) } \\
\quad \text { blue collar }\end{array}$ & 55.6 & 56.3 & $\mathrm{NS}$ \\
$\quad$ white collar & 18.7 & 13.8 & $\mathrm{NS}$ \\
$\quad$ farmer & 2.7 & 4.6 & $\mathrm{NS}$ \\
$\quad$ employer & 6.7 & 10.9 & $\mathrm{NS}$ \\
Immigrants (\%) & 13.8 & 5.2 & $<0.05$ \\
Practising self care (\%) & 68.9 & 58.6 & $<0.05$ \\
Use of alternative care (\%) & 8.9 & 10.3 & $\mathrm{NS}$ \\
Use of analgesics without prescription & 62.2 & 48.3 & $<0.05$ \\
Use of analgesics with prescription & 59.6 & 35.6 & $<0.01$ \\
Symptoms (\%) past three months: & & & $<0.01$ \\
$\quad$ depression & 48.4 & 30.5 & $<0.05$ \\
$\quad$ insomnia & 40.9 & 27.0 & $<0.05$ \\
$\quad$ nervousness & 22.7 & 11.5 & NS \\
Widespread pain (\%) & 44.4 & 34.5 & $<0.01$ \\
Reduced ADL capacity (\%) & 13.8 & 12.1 & $\mathrm{NS}$ \\
\hline Sick leave (\%) (at least one day past three months) & 52.9 & 15.5 & \\
Unemployment (past five years) & 14.6 & 12.6 & \\
\hline
\end{tabular}

summary of multiple tables was used. Correlations between different types of care and medication and hypothetical explanatory variables were expressed as Spearman rank correlation coefficients.

Variables with a significant $(\mathrm{p}<0.01)$ correlation were then used in multiple logistic regression models (forward method) with different types of care and medication as the dependent variables. Variables included in the analysis were dichotomised using dummy variables except age, socioeconomic level, and social network, which were categorised in the analysis. The dummy variable "high pain intensity" was set to one if high pain intensity was reported in any location. The results were expressed as odds ratios (ORs).

The level of significance was set to 0.05 except for the correlations where the level of significance was 0.01 because of the large number of tests. All statistical procedures were computed with SPSS for Windows 6.1.

\section{Results}

SELF CARE

Among people reporting chronic pain $58.2 \%$ had on their own taken steps to reduce the pain, and if self medication was included $73.1 \%$ performed some activity. No gender difference was found but younger people were more prone to practise self care. The most common steps to alleviate pain experience were the use of different types of heat $(34 \%)$ and resting (32\%). Physical activity (walking, jogging, swimming) was used by $21 \%$, and $16 \%$ had changed their working conditions. About $6 \%$ of people reporting pain had used fasting or health food while $1 \%$ had increased their alcohol and tobacco consumption for pain relief.

PRIMARY HEALTH CARE (PHC)

Among people reporting chronic pain $45.7 \%$ had consulted a doctor during the past three months. The inclination for consulting rose with increasing age up to the age group of 55 to 64 but was lower for the oldest age group. The proportion of attenders to PHC with chronic
KEY POINTS

- Chronic pain has a great impact on the use of PHC and analgesics.

- There is an obvious overlap between the use of conventional and alternative care.

- Pain intensity, age, and ethnicity affects the use of self care and PHC.

- Use of analgesics is affected by gender, pain intensity, social network, and use of care.

pain also increased by age except for the age group of $65-74$. Of the people reporting chronic pain $39.5 \%$ attended a PHC doctor during the past three months compared with $25.5 \%$ of people without persistent pain (figures standardised by age, $\mathrm{p}<0.05$ ). In a comparison between gender, independent of age, no difference was found (men $38.5 \%$, women $41.7 \%$ NS.). The calculated number of visits per person to district physicians during the past three months was 2.8 for women and 2.7 for men (NS.). Subjects with a shorter duration of pain (3-6 months) had more PHC contacts $(59 \%)$ compared with those with a longer duration( $>6$ months; $34 \%$ ).

Among people with chronic pain $7.2 \%$ reported at least one visit to a physiotherapist compared with $1.2 \%$ without chronic pain $(p<0.05)$. Attending women reported more visits per person during three months compared with attending men (4.2 versus 3.7 , $\mathrm{p}<0.05$ ) but when comparing the number of attending persons no gender difference was found.

A comparison between visitors and nonvisitors to PHC was undertaken among people with chronic pain reporting high pain intensity (grade 4-5) (table 1). No differences by age, gender, education, and socioeconomic status was found, but immigrants and people practising self care were more often visitors. People with depression, insomnia, general nervousness, and widespread pain were overrepresented among visitors. Sick leave in the past three months, but not reduced ADL capacity, was more frequent in the group of attenders. Visitors more often used analgesics, both with and without prescription.

HOSPITAL CARE

At least one visit to the hospital clinics in the past three months was reported by $12.3 \%$ of people with chronic pain. One fifth of these $(2.8 \%)$ had an emergency contact during the period. The possibility of a visit increased with age but no gender difference was found. Among people without pain $7.3 \%$ reported a visit to the hospital during the same time, a difference not significant when compared with people with chronic pain.

ALTERNATIVE CARE

During the three month period $5.9 \%$ of the people with chronic pain reported use of alternative care. Most visits were to the chiropractor $(3.7 \%)$ and for acupuncture (1.7\%). A few people attended homeopathy, zone therapy, 


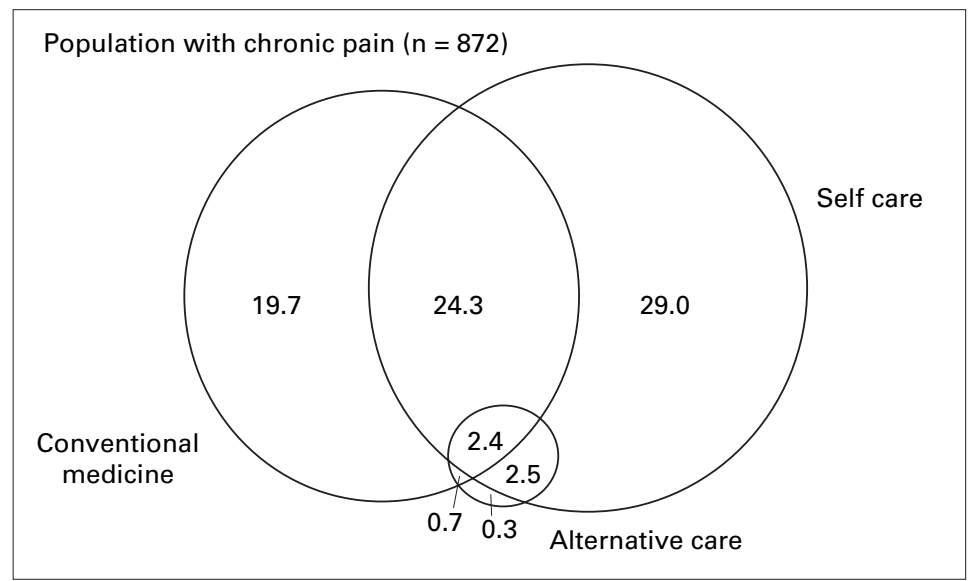

Figure 1 Distribution (\%) of self care and health care contacts in the past three months taken by people with chronic pain in all locations.

Table 2 Odds ratios (95\% confidence intervals) of explanatory variables for self care, consultations of alternative care, and with a physician as dependent variables. Results from logistic regression models among people reporting chronic pain

\begin{tabular}{|c|c|c|c|}
\hline Explanatory variables & Self care & Alternative care & Physician visit \\
\hline \multicolumn{4}{|l|}{ Age } \\
\hline $25-34$ & 1.00 & 1.00 & 1.00 \\
\hline $35-44$ & $1.30(0.71,2.35)$ & $0.88(0.30,2.58)$ & $2.12(1.19,3.78)$ \\
\hline $45-54$ & $0.65(0.36,1.17)$ & $1.47(0.50,4.27)$ & $1.72(0.96,3.06)$ \\
\hline $55-64$ & $0.46(0.26,0.83)$ & $0.89(0.27,2.86)$ & $2.66(1.44,4.89)$ \\
\hline $65-74$ & $0.36(0.18,0.73)$ & $0.50(0.10,2.72)$ & $2.30(1.08,4.93)$ \\
\hline \multicolumn{4}{|l|}{ Socioeconomic level } \\
\hline blue collar & 1.00 & 1.00 & 1.00 \\
\hline white collar & $1.10(0.72,1.69)$ & $0.97(0.35,2.27)$ & $1.87(1.23,2.84)$ \\
\hline farmer & $0.51(0.20,1.33)$ & $2.13(0.44,10.5)$ & $1.47(0.59,3.65)$ \\
\hline employer & $0.66(0.38,1.16)$ & $1.17(0.38,3.62)$ & $0.98(0.56,1.70)$ \\
\hline Immigrant & $1.83(1.01,3.32)$ & $\star$ & $2.30(1.30,4.07)$ \\
\hline Regular physical excercise & $2.59(1.79,3.74)$ & $1.16(0.57,2.38)$ & $1.10(0.76,1.58)$ \\
\hline High pain intensity & $2.78(1.92,4.01)$ & $3.12(1.43,6.79)$ & $1.76(1.24,2.51)$ \\
\hline Feeling depressed & $1.04(0.70,1.54)$ & $1.14(0.56,2.33)$ & $1.72(1.17,2.52)$ \\
\hline Diagnose of chronic disease & $1.37(0.93,2.01)$ & $0.96(0.46,2.00)$ & $1.48(1.04,2.13)$ \\
\hline Self care activities & - & $2.70(1.10,6.62)$ & $1.30(0.90,1.88)$ \\
\hline
\end{tabular}

^Too few people to permit meaningful calculations.

and other naturopathy. Among people without chronic pain $1.2 \%$ (NS compared with chronic pain people) reported use of naturopathy, preferably homeopathy.

FACTORS AFFECTING REPORTS OF SELF CARE AND CONSULTATIONS

From the descriptive results above we found that self care and different types of consultations among people with chronic pain partially overlapped (fig 1). To correct for possible interactions, a multivariate analysis was performed. After an explanatory bivariate correlation analysis, a multiple logistic regression model was created for each of self care, alternative care, and physician consultation (PHC) as the dependent variable (table 2).

People with self care were more likely to have regular physical exercise (OR 2.59), high pain intensity (OR 2.78), and to be immigrants (OR 1.83).

The consultation rate among people with chronic pain with physicians was related to socioeconomic level and ethnicity. The white collar workers reported consultations more often than blue collar workers (OR 1.87). Subgroup analysis showed that white collar workers without high school or university education were most represented (OR 3.91). Immigrants and people with immigrated parents were more likely, compared with natives, to have had a consultation in the past three months (OR 2.30).

Predictory individual factors for consultation were high pain intensity (OR 1.76), depressive symptoms (OR 1.72), and increasing age.

When a comparable analysis was carried out among people without chronic pain, only depressive symptoms (OR 1.80) and presence of a chronic disease (OR 2.58) contributed to the model.

Explanatory variables for use of alternative care were high pain intensity (OR 3.12) and self care activities (OR 2.70).

\section{MEDICATION}

In the studied population $62.4 \%$ (men $54.5 \%$, women $70.1 \% ; \mathrm{p}<0.05$ ) had used analgesics in the past two weeks. No difference by age was found. The use of analgesics with and without prescription and herbal remedies varied between different pain locations. People reporting widespread pain used more analgesics, sedatives, and ointments than people with low back and neck-shoulder pain. When data were standardised for differences in pain intensity, the difference disappeared. For all pain locations women accounted for a higher consumption of analgesics without prescription, and prescribed sedatives.

In the logistic regression model a set of explanatory variables was tested for the outcome of the main groups of medication (table 3). Female gender and sleeping disturbances

Table 3 Odds ratios (95\% confidence intervals) of explanatory variables for medication in the past two weeks with analgesics without prescription, prescripted analgesics and sedatives, and herbal remedies/ointments. Results from logistic regression models among people reporting chronic pain

\begin{tabular}{llll}
\hline Explanatory variables & $\begin{array}{l}\text { Analgesics without } \\
\text { prescription }\end{array}$ & $\begin{array}{l}\text { Analgesics, sedatives with } \\
\text { prescription }\end{array}$ & $\begin{array}{l}\text { Herbal remedies, } \\
\text { ointments }\end{array}$ \\
\hline $\begin{array}{l}\text { Female gender } \\
\text { Socioeconomic level }\end{array} \quad 3.15(1.98,5.03)$ & $1.25(0.73,2.12)$ & $0.64(0.36,1.15)$ \\
$\quad$ blue collar & 1.00 & 1.00 & 1.00 \\
$\quad$ white collar & $1.33(0.66,2.67)$ & $0.83(0.38,1.82)$ & $0.32(0.12,0.80)$ \\
$\quad$ farmer & $0.65(0.17,2.51)$ & $12.1(2.13,68.8)$ & $0.69(0.13,3.59)$ \\
$\quad$ employer & $0.73(0.31,1.74)$ & $1.31(0.49,3.52)$ & $0.24(0.05,1.09)$ \\
Regular physical excercise & $0.64(0.39,1.05)$ & $1.65(0.95,2.84)$ & $1.18(0.67,2.10)$ \\
Intense social network & $0.75(0.34,1.64)$ & $0.38(0.19,0.78)$ & $1.05(0.41,2.68)$ \\
High pain intensity† & $0.84(0.52,1.40)$ & $2.50(1.47,4.26)$ & $2.69(1.51,4.79)$ \\
Insomnia & $1.63(0.99,2.68)$ & $1.68(0.99,2.84)$ & $1.04(0.58,1.86)$ \\
Diagnose of chronic disease & $0.82(0.49,1.37)$ & $2.12(1.25,3.61)$ & $1.30(0.72,2.33)$ \\
Self care activities & $1.23(0.74,2.03)$ & $1.95(1.10,3.45)$ & $2.70(1.45,5.03)$ \\
District physician (visit past three months) & $0.98(0.59,1.60)$ & $2.68(1.59,4.50)$ & $0.66(0.36,1.18)$ \\
Alternative care (visit past three months) & $6.74(1.68,27.1)$ & $1.80(0.55,6.00)$ & $2.28(0.91,7.35)$ \\
\hline
\end{tabular}

${ }^{\star}$ Network score $>4$ on a 7 step scale. $†$ Pain intensity $>3$ on a 5 step scale. 
had an explanatory value for consumption of unprescribed analgesics. High pain intensity and report of self care activity were important in explaining the use of prescribed analgesics, sedatives, and ointments/herbal remedies. An intense social network was associated with low use of prescribed analgesics and sedatives. The group of farmers showed a high odds ratio for prescribed analgesics and sedatives, while white collar workers used herbal remedies and ointments to a lesser extent.

\section{Discussion}

CARE SEEKING

A major aim of this study was to analyse which factors are the most important in explaining care seeking and medication among people with a report of chronic pain. The definition of chronic pain was based on recurrent or persistent pain for more than three months, a time limit supported by an earlier study. ${ }^{1}$ We found that people with chronic pain often reported overlapping activities with the purpose of pain relief. Our study also emphasised the impact of perceived chronic pain on care seeking. Alternative care and also traditional consultations with district physicians and physiotherapists were more common among people reporting chronic pain in comparison with people without chronic pain. A comparable impact of chronic pain was not found on consultations of hospital care. PHC proved to be the type of care mostly used. Visits to PHC and the proportion of attenders to $\mathrm{PHC}$ with chronic pain increased by age to a maximum at the age of 55-64. The prevalence of chronic pain by age and gender has in several studies a similar distribution with prevalence maxima in the middle ages for both genders, the women being slightly younger. ${ }^{124}$ These results indicate that the presence of chronic pain is accompanied by a similar pattern of the use of PHC. The further logistic regression analysis revealed that not only the presence but also the intensity of pain was important in clarifying the use of care. The multivariate analysis also demonstrated that age, socioeconomic level and ethnicity were important factors for both self care and care seeking.

The finding that there is a correlation between the consumption of health care and chronic pain is known also from other settings. ${ }^{75}$ Our study, however, demonstrated the close linkage between not only the prevalence of chronic pain but also the pain intensity independent of pain location and depressive symptoms. According to the Health Belief model ${ }^{8}$ one of the four important factors explaining care seeking behaviour is perceived severity of symptoms. The other contributory factors are perceived vulnerability to illness, perceived costs, and perceived benefits of action. The vulnerability to illness has been related in other studies to general anxiety and worry of symptoms ${ }^{16}$ but also to an external locus of control. ${ }^{17}$

Depressive symptoms among people with chronic pain contributed in our study to care seeking. Either depression is primary or secondary to chronic pain, experience of worry may increase the vulnerability to illness and hence care seeking. In the American study by Von Korff et al pain severity, persistence and recency of the onset of pain all increased the likelihood of a health care contact. They found that psychological distress, measured by depression and anxiety (SCL-90), did not increase the probability of a health care contact, but distressed persons reported more often pain in multiple locations. Several psychological items from our symptom list (depression, anxiety, increased tension) correlated with visits to physicians. Because of a high correlation between the factors, only depression had an explanatory value in the regression model.

Increasing age, but surprisingly not gender, explained some variation in physician visits. However these findings are supported by an American study of delimited pain syndromes (back pain, headache, chest pain, abdominal pain, and temporomandibular pain). ${ }^{7}$ In that study, multivariate analysis showed a small effect of age from the age of 45 on ambulatory care visits but no association by gender. Musculoskeletal symptoms in a diary study ${ }^{16}$ also resulted in a higher probability of care for men. In general it has been demonstrated that lower socioeconomic groups correlate with an increased probability of contacts with a physician. In contrast self care was practised by fewer people in lower than in upper social groups. ${ }^{18}$

Our findings with a raised odds ratio (1.87) for white collar persons regarding visits to physicians are indirect supported by an interview study of perceived illness in relation to socioeconomic level and primary care. ${ }^{19}$ In that study an increased morbidity among manual workers was not matched for men by a difference in GP consultation rates. These unequal distributions of visits could reflect differences in the perception of pain symptoms as a health problem. A person used to a low physical work strain and no "everyday" experience of tense or aching muscles may react more intensely and with a greater need for care because of longstanding pain symptoms. Higher education has also been attributed importance as a factor in increased use of care because of more active coping strategies. ${ }^{16}$ According to the Health Belief model, differences in perceived costs and benefits of action may explain the difference. ${ }^{8}$ In studies from the USA in the 1980s economic inequality between socioeconomic groups explained reduced rates of attendance..$^{20}$ The cost of a visit to a Swedish district physician at the time of the study was moderate (about $£ 10$ ) but could still be an important factor in explaining the socioeconomic variation.

We found that immigrants with chronic pain in Bromölla and Simrishamn revealed a higher probability of performing self care as well as visiting a physician compared with native people. Immigrants in our population originated mainly from the Mediterranean part of Europe, a group previously shown to have a raised level of health care contacts. ${ }^{21}$ Ethnicity has also been shown to be a predictor of 
both musculoskeletal morbidity ${ }^{22}$ and early retirement. ${ }^{23}$ Pain symptoms and their behavioural responses are influenced by the cultural background $^{5}$ and this could be expected partly to explain differences in care seeking.

We lack information as to whether self care activities preceded care seeking, but they often coincided. More than half of the people reporting chronic pain stated self care but the major actions were rather passive (rest, heat). No difference by socioeconomic level was found. Similar patterns of action were shown in a Danish study of self care of different symptoms. ${ }^{18}$ In that study $83 \%$ of the people with lumbar pain reported self treatment activities consisting of rest, avoiding of lifting and use of heat pads, and only $20 \%$ used medication. An important finding of our study was that the use of self care or naturopathy did not imply a lower contact rate with conventional medicine. People using self care also had more health care contacts and used more prescribed analgesics and ointments compared with those performing no self care. Another Scandinavian study of care seeking ${ }^{24}$ concluded that self care of all kinds of illness was associated with lowered use of PHC. The question about self care in that study was however hypothetical and not associated with a symptom.

Compared with other data, ${ }^{10}$ contacts with both physiotherapist and alternative care were uncommon among people with chronic pain in our population. At the time of the study physiotherapists in the area were not trained to use acupuncture and together with the limited access to these care givers in the rural districts the low figures could possibly be explained. The access to PHC doctors was on the contrary fairly good (2500 inhabitants per doctor) making consulting data comparable to other studies. ${ }^{6}$

\section{MEDICATION}

The use of analgesics was the most common action undertaken by people with chronic pain. In the studied population $62.4 \%$, with a female predominance $(70.1 \%$ versus $54.5 \%)$, had used analgesics during the past two weeks. A comparison with data from a general Swedish population is possible from a study that used the same question on medication and recall period. ${ }^{12}$ In that study $34.7 \%$ used analgesics also with a larger proportion of women $(42.4 \%)$ compared with men $(26.8 \%)$. Thus, the report of chronic pain correlates to a substantial increase with the consumption of analgesics. A Norwegian study of a general population aged 12-56 showed lower figures: $28.1 \%$ of women and $12.8 \%$ of men had used analgesics in the previous two weeks. ${ }^{9}$ The lower figures may be because of different age distribution but also to lower drug use in Norway compared with other countries. ${ }^{12}$ Both these studies support our findings of no age difference in the consumption of analgesics. We found that unprescribed analgesics were used twice as commonly as prescribed ones, and this was true for both genders.

Besides female gender, the use of analgesics was increased when the factors of insomnia, high pain intensity and a low social network were present. Socioeconomic level had a low impact on consumption of analgesics, but white collar people tended to have an increased use of unprescribed analgesics. This fact is also supported by the Swedish study above, ${ }^{12}$ and a hypothetic explanation could be more knowledge of available medication and an increased perceived need for care as was proposed in the part concerning physician visits. Furthermore, this study ${ }^{12}$ showed a predictory value for the use of analgesics in specific pain conditions, insomnia and visits to the physiotherapist. In our study visits to a physician but not to a physiotherapist related to the use of prescribed analgesics and sedatives. The presence of self care and visits of naturopathy were, like high pain intensity, associated with the use of herbal remedies and ointments.

Pain intensity was of significant importance for the intake of prescribed but not for unprescribed analgesics, a fact that could reflect different mechanisms of use. Unprescribed analgesics may be used also for fever, infections, and psychosomatic conditions where the element of pain is low. ${ }^{25}$

The use of muscle relaxants, sedatives, and hypnotics among people with chronic pain was also higher than among a general population. ${ }^{11}$ This could be explained by several factors: more perceived tension and anxiety, more insomnia and depressiveness among people with pain but also the belief of the doctor that these drugs may benefit the pain patient. ${ }^{12}$

The analysis of the influence of gender on health care seeking and medication revealed no difference in district physician visits but a raised female use of physiotherapy as well as of analgesics and sedatives. In contrast with earlier reports ${ }^{10} 18$ we found no gender difference regarding the use of self care and naturopathy. To sum up, the impact of gender in our study was low in contrast with other studies of general care seeking. ${ }^{8}$ Our results also indicate that sick role development and health care behaviour related to chronic pain in themselves have little or no gender difference. These findings are in agreement with a study of gender roles and illness orientation. ${ }^{26}$ Women are more likely to perceive symptoms and have gender specific symptoms ${ }^{16}$ that could explain the increased use of analgesics compared with men. The statement that physicians have a greater tendency to prescribe drugs to female patients ${ }^{9}$ was, in our study, not supported by the low odds ratio (1.25) of prescribed drugs in contrast with unprescribed analgesics (3.15).

The strength of our survey is the coverage of all types of health care and medication reported by the respondents and a high, and evenly distributed, response rate. ${ }^{1}$ The selection bias was small because respondents were randomly selected from population registers in defined areas. An inherent difficulty of this type of study is recall bias for various questions. Recall time in our questions ranged from two weeks to three months, which may influence the responses in different ways. Studies on methodology ${ }^{27}$ have shown both over-reporting and under-reporting according to type of 
question and respondent. However, a memory time of two weeks for medication has been tested successfully in earlier studies. ${ }^{12}$ For visits and symptoms a recall time of three months gave valid estimations. ${ }^{13}$ Interpretation of the survey results must be done with care especially concerning casual relations. We want also to emphasise that the analyses are based on aggregated data and specific visits cannot be connected to individual people. Consequently the results must be used and generalised on a group level.

In conclusion, we found that chronic pain has an impressive impact on PHC utilisation and medication. Different actions of health care obviously overlapped. Beside the need for pain relief several other factors such as socioeconomic level, network, and symptoms such as depression and insomnia influenced the pattern of health care utilisation and medication. High pain intensity was a strong predictor of all types of health care behaviour including medication.

Reducing pain experience and treatment of coincident depression and insomnia are important issues in improving the situation among people with chronic pain. Acupuncture, physiotherapy, and thereby transcutaneous electrical nerve stimulation were poorly utilised in our study compared with drug use. An increased use of these methods of non-medication pain relief may be beneficial in the PHC work with chronic pain patients. Planning of resources and knowledge of PHC must be influenced by the high prevalence and attendance rate of individuals with chronic pain.

Funding: we are grateful for financial support from Kristianstad County Council and AB Astra, Sweden.

Conflicts of interest: none.

1 Andersson HI, Ejlertsson G, Leden I, et al. Chronic pain in a geographically defined general population: studies of differences in age, gender, social class, and pain localization. Clin F Pain 1993;9:174-82.

2 Brattberg G, Thorslund M, Wikman A. The prevalence of pain in a general population. The results of a postal survey pain in a general population. The results of a

3 Ballina Garcia FJ, Hernandez Mejia R, Martin Lascuevas P, et al. Epidemiology of musculoskeletal complaints and use et al. Epidemiology of musculoskeletal complaints and use
of health services in Asturias, Spain. Scand 7 Rheumatol 1994;23:137-41.
4 Potter RG, Jones JM. The evolution of chronic pain among patients with musculoskeletal problems: a pilot study in patients with musculoskeletal problems: a pil
primary care. $B r \mathcal{F}$ Gen Pract 1992;42:462-64.

primary care. Br $\mathcal{F}$ Gen Pract 1992;42:462-64.
5 Bates MS, Edwards WT. Ethnic variations in the chronic pain experience. Ethn Dis 1992;2:63-83.

6 Rekola KE, Keinanen-Kiukaanniemi S, Takala J. Use of primary health services in sparsely populated country districts by patients with musculoskeletal symptoms: consultations with a physician. F Epidemiol Community Health 1993;47: 153-7.

7 Von Korff M, Wagner EH, Dworkin SF, et al. Chronic pain and use of ambulatory health care. Psychosom Med 1991;53:61-79.

8 Campbell SM, Roland MO. Why do people consult the doctor? Fam Pract 1996;13:75-83

9 Eggen AE. Pattern of drug use in a general populationprevalence and predicting factors: the Tromso study. Int $\mathcal{F}$ Epidemiol 1994;23:1262-72.

10 Thomas KJ, Carr J, Westlake L, et al. Use of non-orthodox and conventional health care in Great Britain. BMF 1991; 302:207-10

11 Wessling A. Continuous recording of drug prescribing in Sweden 1974-1983. Methods and examples of utilization of data. Eur f Clin Pharmacol 1987;33:7-13.

12 Antonov K, Isacson D. Use of analgesics in Sweden-the importance of sociodemographic factors, physical fitness, health and health-related factors, and working conditions. Soc Sci Med 1996;42:1473-81.

13 Statistics Sweden. Living conditions. Appendix 13. Technical report for 1984-85, 1986-1987, and 1988-1989 years surveys of living conditions. Örebro: SCB-Tryck, 1991.

14 Nyberg P. Social networks and solution of problems. [In Swedish]. Lund: Health Sciences Centre, Dalby, 1987.

15 Egan KJ, Katon WJ. Responses to illness and health in chronic pain patients and healthy adults. Psychosom Med 1987;49:470-81

16 Verbrugge LM, Ascione FJ. Exploring the iceberg. Common symptoms and how people care for them. Med Care 1987; 25:539-69.

17 Ingham JG, Miller PM. Self-referral to primary care: symptoms and social factors. $\mathcal{F}$ Psychosom Res 1986;30:49-56.

18 Dean KJ, Holst E, Wagner MG. Self-care of common illnesses in Denmark. Med Care 1983;21:1012-32.

19 Bucquet D, Curtis S. Socio-demographic variation in perceived illness and the use of primary health care: the value of community survey data for primary care service planning. Soc Sci Med 1986;23:737-44.

20 Yelin E, Bernhard G, Pflugrad D. Access to medical care among persons with musculoskeletal conditions. A study using a random sample of households in San Mateo County, California. Arthritis Rheum 1995;38:1128-33.

21 Ektor Andersen J, Janzon L, Sjolund B. Chronic pain and the sociodemographic environment: results from the Pain Clinic at Malmo General Hospital in Sweden. Clin f Pain 1993;9:183-8.

22 Sundquist J, Rosén U, Johansson SE. Ethnicity as a risk indicator for musculoskeletal disease, long consultation imes and care utilization. Scand F Soc Welfare 1994;3:21217

23 Edén L, Ejlertsson G, Lamberger B, et al.Immigration and socio-economy as predictors of early retirement pensions. Scand F Soc Med 1994;22:187-93.

24 Grimsmo A, Siem H. Factors affecting primary health care utilization. Fam Pract 1984;1:155-61.

25 Bush PJ, Rabin DL. Who's using nonprescribed medicines? Med Care 1976;14:1014-23

26 Hibbard JH, Pope CR. Gender roles, illness orientation and use of medical services. Soc Sci Med 1983;27:129-37.

27 Roberts RO, Bergstralh EJ, Schmidt L, et al. Comparison of self-reported and medical record health care utilization measures. F Clin Epidemiol 1996;49:989-95. 